\title{
NUMERICAL AND EXPERIMENTAL STUDY OF PRODUCING TWO-STEP FLANGES BY EXTRUSION WITH A MOVABLE SLEEVE
}

\begin{abstract}
The paper presents a new metal forming process for producing two-step external flanges on hollow parts. With this method, the flange is extruded by a movable sleeve, which moves in the opposite direction to the punch. This reduces the phenomenon of buckling of the tube wall, which allows extruding flanges with relatively large volumes. The new method was applied to produce a two-step flange on the end of a tubular billet made of 6060 aluminum alloy. This cold metal forming process was designed based on numerical simulations and experimental tests. The effect of the basic technological parameters on metal flow was investigated and limitations of the process were identified. The experimental results confirmed the possibility of forming a two-step flange with a diameter that is approximately twice as big as the external diameter of the tubular billet.

Keywords: hollow parts; flange forming; injection forging; radial extrusion
\end{abstract}

\section{Introduction}

Hollow axes and shafts are often used in machine design as drive shafts. These components mainly carry torsional and bending stresses. Given their weight, hollow components are more favorable to use than solid elements. For example, a hollow shaft with an outside diameter which is bigger by $20 \%$ and an inside diameter that is smaller by $3 \%$ than those of a solid shaft is not only half lighter than the solid one but also has the same torsional strength as the solid shaft. Taking it into consideration, the use of hollow components is desired as it enables significant reduction in structure weight, which is cost effective.

Hollow shafts are mainly manufactured by metal forming, machining and casting techniques. As far as mass production is concerned, metal forming offers the most advantages due to short manufacturing time, low material waste and high strength properties. Metal forming techniques for producing hollow parts include:

- $\quad$ spinning $[1,2]$,

- $\quad$ standard forging $[3,4]$,

- $\quad$ rotary swaging $[5,6]$,

- $\quad$ extrusion [7],

- $\quad$ rotary compression $[8,9]$,

- flanging [10,11],

- $\quad$ sequential upsetting [12],

- $\quad$ radial extrusion.

Owing to the limitations of the existing metal forming processes for producing hollow parts, a new alternative method has been developed. With this method hollow parts are produced by extrusion with a moving sleeve. This paper reports the results of numerical and experimental investigation of the developed process for forming a two-step flange on the end of a hollow billet. The aim of the study was to determine the effect of some technological parameters on the extrusion process and to define failure modes.

\section{Description of extrusion with a moving sleeve}

A schematic design of the process for forming flanges by extrusion using a moving sleeve is shown in Fig. 1. As a result of upsetting a billet $3 a$ using a punch 1 , metal fills in a closed impression with an initial height $h_{0}$ adjusted by a distance plate 5 . The closed impression is formed by a moving sleeve 2 , a fixed sleeve 4 and the punch. During filling, the moving sleeve moves in a direction opposite to that of the punch. As a result, the volume of the closed impression gradually increases, which enables extruding forgings $3 b$ with flanges described by a height $H$ which is several times bigger than a wall thickness $g$ of the billet.

The proposed process can be performed in two variants depending on how the moving sleeve is driven. One, the sleeve can be set into motion independently of the punch or, two, it can move automatically due to the action of metal load. With enforced movement of the sleeve, the process must be performed on a machine provided with two independent sources of power, e.g. on a three-slide forging press. Such machines, however,

\footnotetext{
* LUBLIN UNIVERSITY OF TECHNOLOGY, FACULTY OF MECHANICAL ENGINEERING, DEPARTMENT OF COMPUTER MODELLING AND METAL FORMING TECHNOLOGIES, NADBYSTRZYCKA 36 STREET, 20-618 LUBLIN, POLAND

\# Corresponding author: g.winiarski@pollub.pl
} 
are not very widely used in the industry. Therefore, this study investigates the proposed extrusion process with an automatically moving sleeve. Here, the punch is the only tool that is driven, so the process can be performed on standard and widely used forging machines such as hydraulic or mechanical presses. To ensure better filling, one can use springs to clamp the moving sleeve. The ends of the springs are fixed to a base 6 and to the moving sleeve.

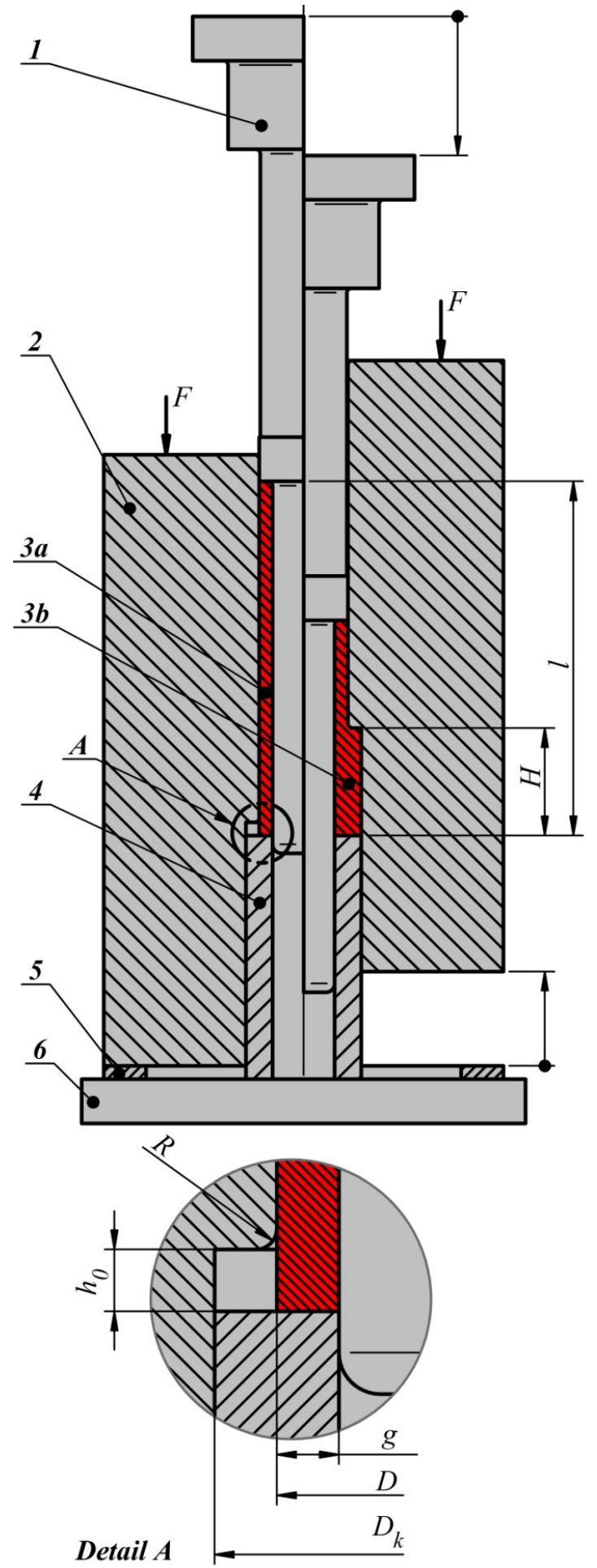

Fig. 1. Schematic design of a process for forming flanges by extrusion with a movable sleeve; 1 - punch, 2 - moving sleeve, $3 a$ - billet, $3 b$ forging, 4 - fixed sleeve, 5 - distance plate, 6 - base

\section{Methodology and the numerical model of the process}

It was assumed that a stepped flange would be formed on the end of a hollow billet by extrusion with a moving sleeve. Large-volume flanges cannot be formed in one operation as this would lead to failure modes. This study involved analysis of the extrusion process with a moving sleeve in terms of the effect of flange diameter and sleeve clamp on the process. The corner radius of the moving sleeve was set to be equal to the billet wall thickness, while the initial displacement of the sleeve was made equal to $1 / 3$ of billet wall thickness $\left(R=g, h_{0}=1 / 3 g\right)$. A list of the examined cases of flange formation is given in Table 1 . The analysis was performed on aluminum alloy 6060 tube sections subjected to annealing prior to cold extrusion. The moving sleeve was clamped by 12 springs, their total modulus of elasticity, determined by the authors' in the previous study, being set to $1039.7 \mathrm{~N} / \mathrm{mm}$.

TABLE 1

Investigated cases of flange extrusion (denoted in accordance with Fig. 1)

\begin{tabular}{|c|c|c|c|c|c|c|c|}
\hline \multirow{2}{*}{ No. } & $\boldsymbol{g}$ & $D$ & I & $h_{0}$ & $R$ & $D_{k}$ & $F$ \\
\hline & $\mathbf{m m}$ & $\mathbf{m m}$ & $\mathbf{m m}$ & mm & $\mathbf{m m}$ & $\mathbf{m m}$ & $\mathbf{N} / \mathbf{m m}$ \\
\hline 1. & \multirow{4}{*}{3} & \multirow{4}{*}{20} & \multirow{4}{*}{80} & \multirow{4}{*}{1} & \multirow{4}{*}{3} & \multirow{2}{*}{26} & 0 \\
\hline 2. & & & & & & & 1039.7 \\
\hline 3. & & & & & & \multirow{2}{*}{29} & 0 \\
\hline 4. & & & & & & & 1039.7 \\
\hline
\end{tabular}

The extrusion process by a moving sleeve was verified numerically using the Deform-3D simulation software [13]. The thermomechanical simulations consisted in modelling a section of the billet described by an angle of $36^{\circ}$ (1/10 of the billet) with a defined symmetry plane. The billet discretization was made using four-node tetragonal elements. The material model of the aluminium alloy was described by equation (1):

$$
\sigma_{p}=147.5 \cdot \varphi^{0.2}
$$

as determined by the authors' in their previous study on compression of cylindrical specimens. The analysis also involved determination of a friction factor between the frictional pair aluminium alloy 6060-tool steel. The friction factor was measured by upsetting a ring-shaped specimen. The numerical analysis was performed without lubrication and with lubrication by molybdenum disulfide applied in the experimental tests. The friction factors determined in the numerical analysis were $m=0.6$ and $m=0.16$. As a result, the friction factor was set to $m=0.16$. The temperature of the tools and billet was $20^{\circ} \mathrm{C}$, and the tool-billet heat transfer coefficient was set to $10 \mathrm{~kW} / \mathrm{m}^{2} \mathrm{~K}$. The punch velocity was maintained at $50 \mathrm{~mm} / \mathrm{min}$. Due to the fact that the modelling was performed for a $1 / 10$ of the billet, the total modulus of elasticity of the springs was reduced by ten times to $103.97 \mathrm{~N} / \mathrm{mm}$. 


\section{Results discussion}

Flanged forgings obtained in the numerical simulations are shown in Fig. 2. It can be observed that the produced parts have defects or are defect-free depending on flange diameter and clamp force on the moving sleeve. Out of four investigated cases, a part without defects is produced only in Case 2, wherein a $26 \mathrm{~mm}$ diameter flange is extruded using a moving sleeve with clamp (Fig. 2b). In other cases, we can observe underfill (details $A$ and $B$ ) or overlap (detail $C$ ). Underfill occurs when a $29 \mathrm{~mm}$ diameter flange is produced, a process wherein the moving sleeve is not clamped by springs. This defect is not observed in other cases.

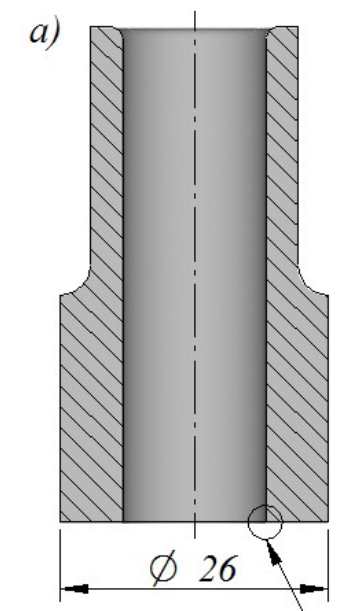

b)
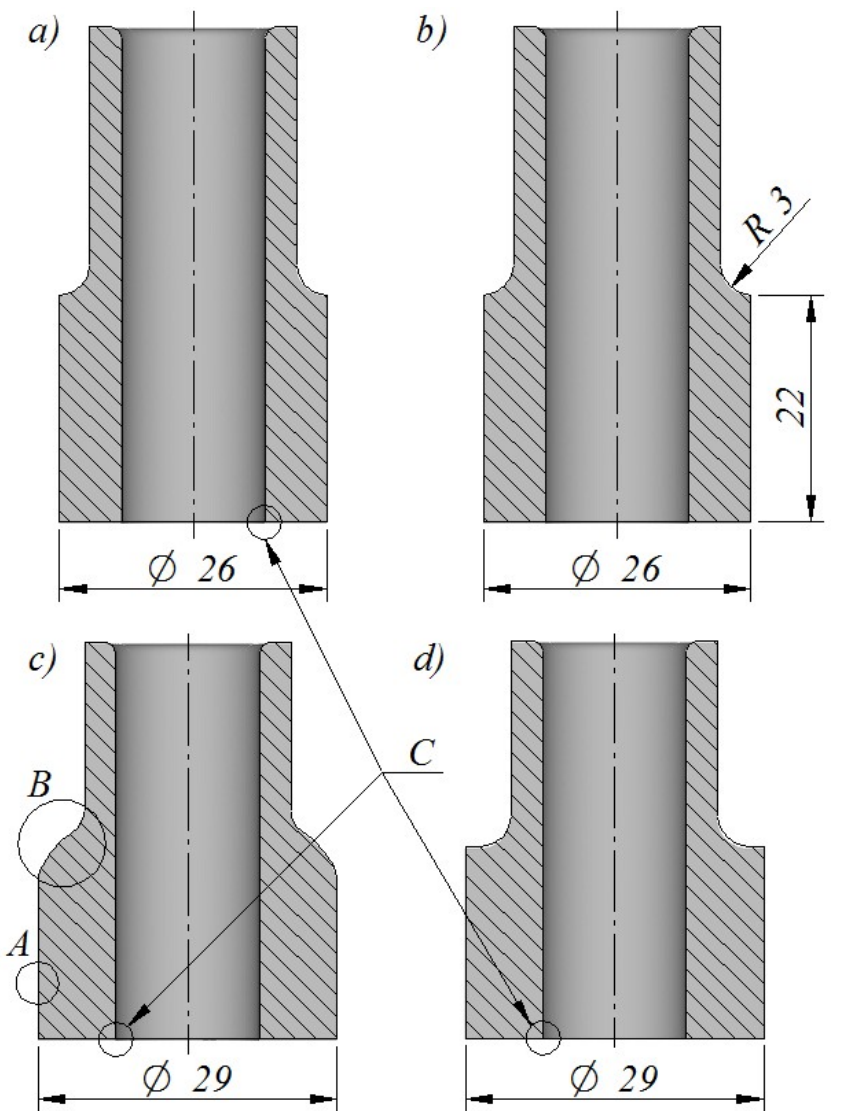

Fig. 2. Forgings in the final stage of extrusion with a moving sleeve; denoted in accordance with Table 1: a) Case 1, b) Case 2, c) Case 3, d) Case 4 .

The numerical results demonstrate that apart from underfill another failure mode in extrusion with a moving sleeve is billet wall buckling in the initial stage of the process, leading to overlap. Considering the four analyzed cases, overlap occurred in three of them. In one case, however, wherein a flange with a diameter of $26 \mathrm{~mm}$ was produced using a clamped moving sleeve (Fig. 2b), the defect did not occur. The regions with overlap are marked in Fig. 2 as detail $C$, while their magnified images are shown in Fig. 3.

Overlap occurs in the initial stage of the process when the moving sleeve begins to move in a direction opposite to that of the punch. Its motion begins before metal has completely filled a)

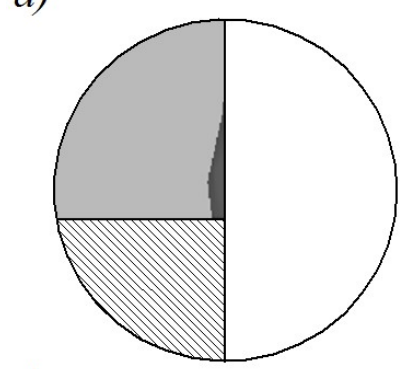

c)

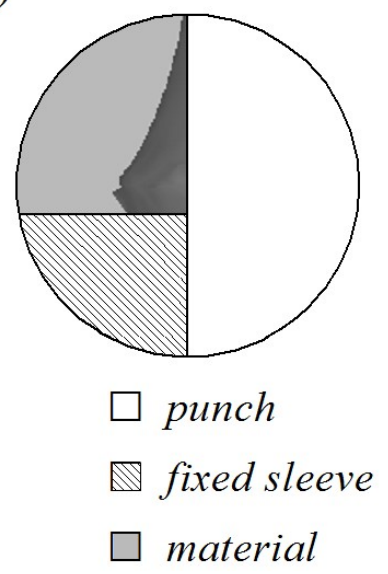

b)

d)
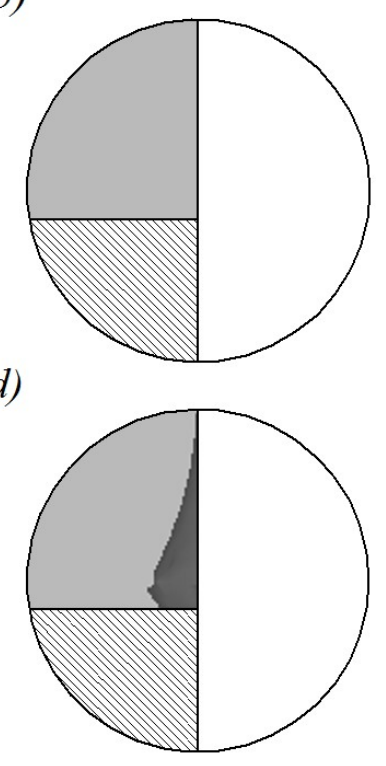

Fig. 3. Forgings at the beginning of extrusion with a moving sleeve; denoted in accordance with Table 1: a) Case 1, b) Case 2, c) Case 3, d) Case 4

in the closed impression with an initial height $h_{0}$. This may lead to billet wall buckling, which, in turn, leads to part defects. The motion of the moving sleeve depends on variations in the forces acting on the sleeve, a simplified diagram of these variations being shown in Fig. 4. The moving sleeve has three characteristic zones. The first zone is the hole with a diameter $D$, the second one is the edge with a corner radius $R$, while the third zone is responsible for forming a flange with a diameter $D_{k}$. Unit pressures exerted on the moving sleeve by the material depending on the degree of advancement of the process lead to the generation of forces in each zone. As a result, we can distinguish resultant forces acting parallel to the axis of the billet. The forces acting in this direction: friction forces and loads, affect motion of the moving sleeve. When the extrusion process is performed with the clamped moving sleeve, it is necessary to take account of the clamp force. The moving sleeve starts to move when the load force $F_{N}$ is higher than the sum of the friction force $F_{T}$ and the clamp force acting on the sleeve $F_{S}$. At the beginning of the extrusion process the workpiece does not contact the impression in the third zone and the produced load force $F_{N}$ relatively quickly becomes higher than the sum of the friction force $F_{T}$ and the clamp force $F_{S}$. The moving sleeve begins to move in the initial stage of the process, which in certain cases - as the numerical results demonstrate - can lead to overlap.

In the examined cases of flange extrusion, the forming force ranges between 40 and $115 \mathrm{kN}$ (Fig. 5). The highest forces can be observed in Case 2, where the produced forgings have the correct shape. The variations in the forces applied to 


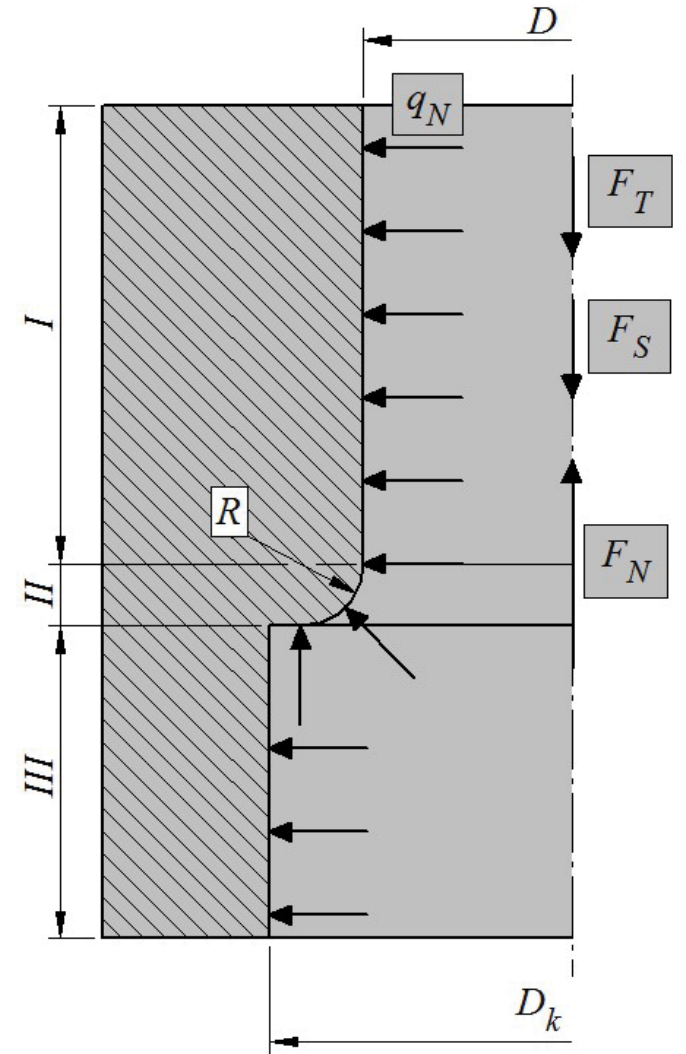

Fig. 4. Simplified schematic diagram of forces and loads acting on the moving sleeve in the extrusion process for flanges

the punch reveal that the application of the clamp force on the moving sleeve leads to an increase in the total forming force. This can be observed for the flange with two diameters: $26 \mathrm{~mm}$ and $29 \mathrm{~mm}$. The variations in the forces in Case 3, in which the

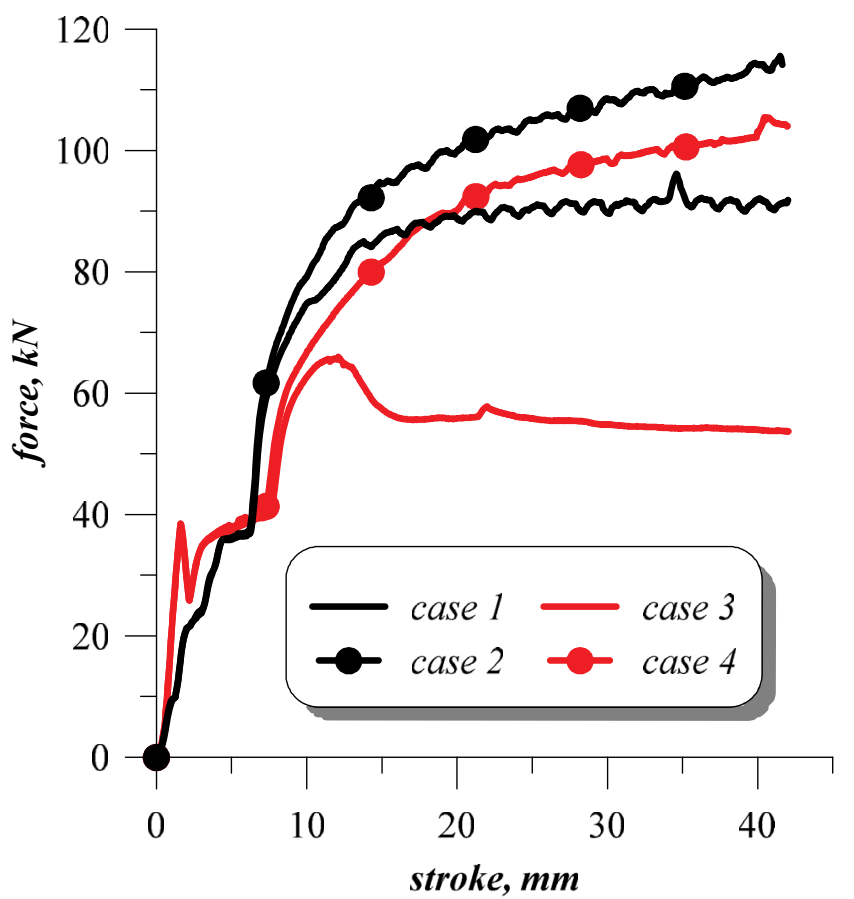

Fig. 5. Variations in punch-applied force versus its displacement for the investigated extrusion cases moving sleeve was not clamped and the flange diameter was set to $29 \mathrm{~mm}$, significantly differ from those observed in other cases. The lower values of the forces are due to underfill.

The results demonstrate that the forgings obtained in Case 2 have the highest quality. Here, the produced flange diameter is bigger than that of the billet by two wall thicknesses $\left(D_{k}=D+2 g\right)$. The moving sleeve with clamp was described by a corner radius equal to the billet wall thickness and had its initial displacement equal to $1 / 3$ of billet wall thickness $(R=g$, $h_{0}=1 / 3 g$ ). This served as a basis for designing the extrusion process for the second step of the flange, the diameter of which was increased while its height was decreased. Assuming that after the first operation the flange diameter is $D_{k 1}=26 \mathrm{~mm}$ and the wall thickness on the flanged billet section is $g_{1}=6 \mathrm{~mm}$, it was analogically assumed that the dimensions obtained after the second operation would be as follows: flange diameter $D_{k 2}=38 \mathrm{~mm}$, corner radius of the moving sleeve $R_{2}=6 \mathrm{~mm}$, and initial displacement $h_{02}=2 \mathrm{~mm}$.

The numerical results given in Fig. 6 illustrate the distributions of effective strains and reduced stresses. Both the highest strains and reduced stresses are located in the region of the two-step flange. Here, the strains can reach up to 5 and the reduced stresses up to $230 \mathrm{MPa}$. In other regions of the forging, the material is not deformed; it only moves downwards along the punch. Therefore, the strains and stresses in this region are insignificant.

a)

b)

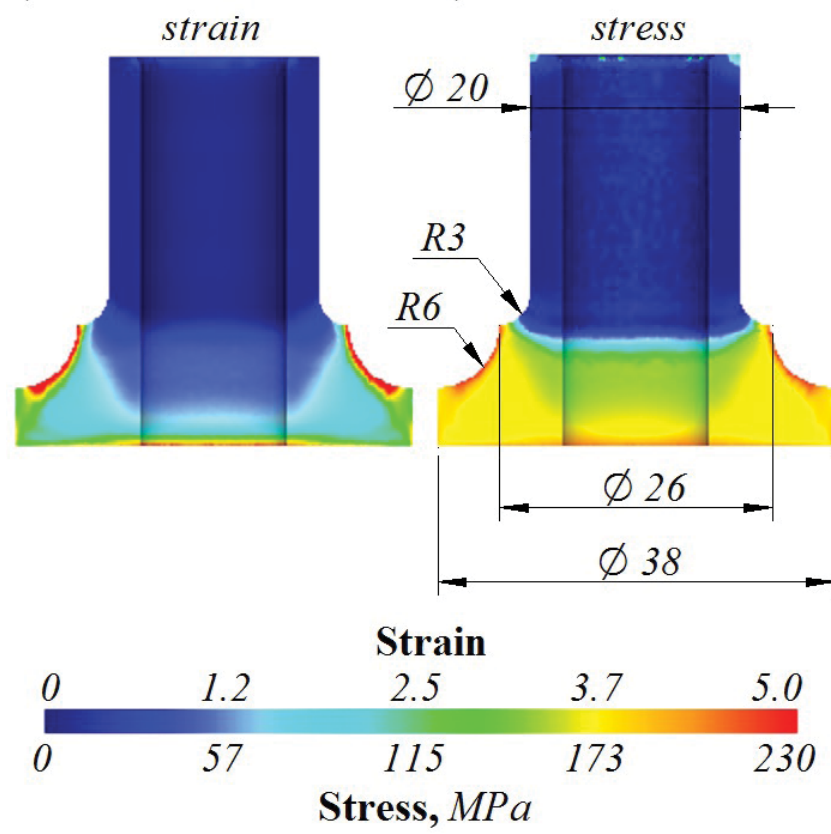

Fig. 6. Distributions of: a) effective strains, b) reduced stresses in the forging with a two-step flange

Forgings produced in the experiments are shown in Fig. 7. The extrusion process for a two-step flange involved interoperation annealing which followed the extrusion of a single-step flange. The produced parts are correctly shaped and free from defects such as overlap or underfill. 
a) b)

c)

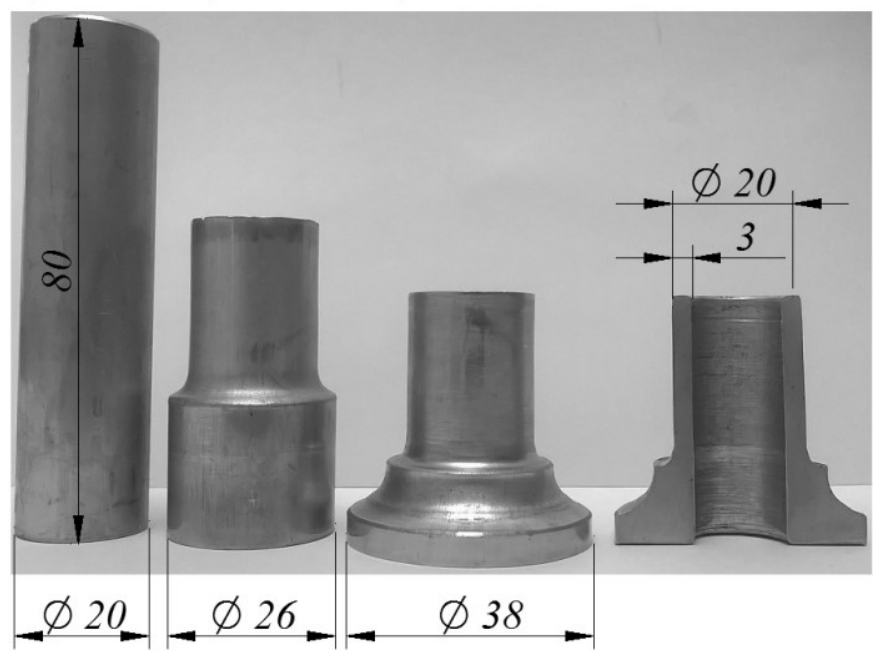

Fig. 7. Forgings produced in experimental tests: a) billet, b) one-step flange, c) two-step flange

The proposed method of extrusion using a moving sleeve can be used to form flanges with relatively large volumes. The volume of the two-step flange produced in the experiments is $V_{1}=9132.6 \mathrm{~mm}^{3}$ (Fig. 8a), while the billet volume is $V=12817.6 \mathrm{~mm}^{3}$. As a results, over $70 \%$ of the billet was formed into a flange.

To compare the proposed method with other techniques for forming flanges, we also determined maximum dimensions of a flange produced by radial extrusion. It was found that the flange thickness is constrained by billet wall buckling in the initial stage of the process, whereas increasing its diameter can lead to cracking. The experimental results demonstrate that the maximum thickness and diameter of the flange are $2 \mathrm{~mm}$ and $47 \mathrm{~mm}$, respectively, which means that the flange volume is $V_{2}=4005.5 \mathrm{~mm}^{3}$, which is approx. $30 \%$ of billet a)

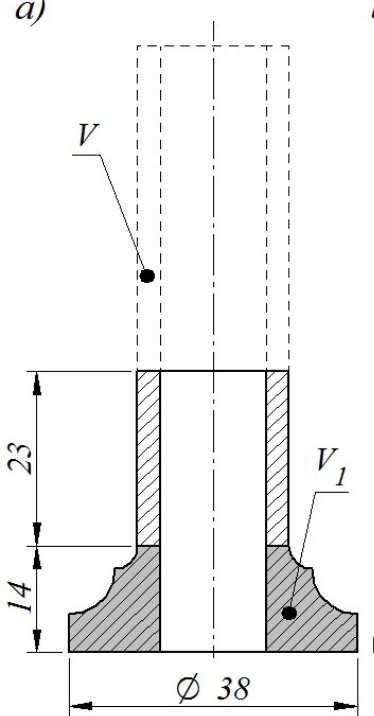

b)

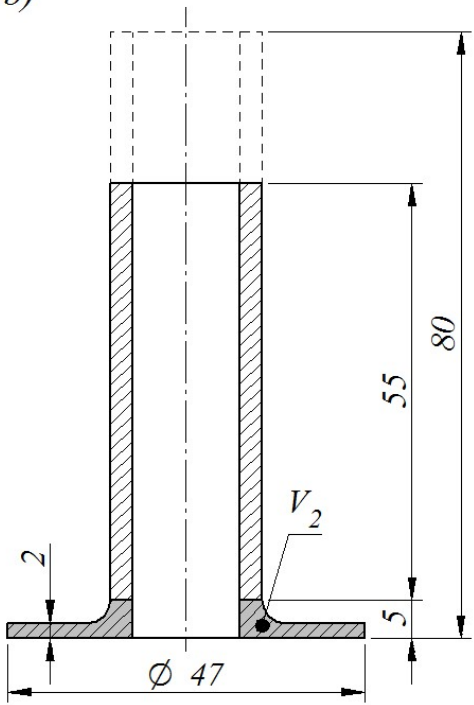

volume (Fig. 8b). The results of the analysis of volume of the flanges produced by the two described methods demonstrate that the flange produced by extrusion using a moving sleeve is over twice as big as that produced by radial extrusion $\left(V_{1}=2.28 V_{2}\right)$.

\section{Summary and conclusions}

The numerical results and experimental findings confirm that flanges can be formed in hollow parts by with using a moving sleeve. The method enables manufacturing flanges with relatively large volumes. A significant amount of metal undergoes deformation due to the moving sleeve which moves in a direction which is opposite direction to that of the punch. The results demonstrate that a certain setting of the sleeve can prevent failure modes such as billet wall buckling leading to overlap and underfill. An important element of the process is the clamp force exerted on the moving sleeve because it prevents the sleeve from moving too fast and, hence, from underfill and overlap. Another important aspect of the proposed technique is that parts can be produced in two stages, which enables forming flanges with a diameter that is nearly twice as big as the initial diameter of the billet.

\section{REFERENCES}

[1] O. Music, J.M. Allwood, K. Kawai, J. Mater. Process. Tech. 210 (1), 3-23 (2010).

[2] M.J. Davidson, K. Balasubramanian, G.R.N. Tagore, J. Mater. Process Tech. 203, 321-325 (2008).

[3] P. Skubisz, Ł. Lisiecki, J. Sińczak, Metalurgija 54 (2), 339-342 (2015).

[4] M. Wolfgarten, D. Rosenstock, L. Schaeffer, G. Hirt, Metall. Ital. 4, 43-49 (2015).

[5] S.J. Lim, H.J. Choi, C.H. Lee, J. Mater. Process Tech. 209 (1), 283-288 (2009).

[6] R. Hebdzyński, S. Kajzer, R. Kozik, J. Mater. Process Tech. 64, 199-206 (1997).

[7] G.C. Wang, G.Q. Zhao, X.H. Huang, Y.X Jia, J. Mater. Process Tech. 121, 259-264 (2002).

[8] Z. Pater, J. Tomczak, T. Bulzak, Metalurgija 54 (2), 419-422 (2015).

[9] J. Tomczak, Z. Pater, T. Bulzak, Int. J. Adv. Manuf. Tech. 82, 1281-1291 (2016).

[10] G. Winiarski, A. Gontarz, Z. Pater, Arch. Civ. Mech. Eng. 15 (4), 911-916 (2015).

[11] X.M. Qiu, L.H. He, J. Gu, X.H. Yu, Thin Wall. Struct. 80, 32-37 (2014).

[12] X.L. Hu, Z.R. Wang, J. Mater. Process Tech. 151, 321-327 (2004).

[13] A. Dziubińska, A. Gontarz, Metalurgija 54 (3) 555-558 (2015).

Fig. 8. Dimensions of flanged forgings produced by: a) extrusion with a moving sleeve, b) radial extrusion 